\title{
Ignoring genotype by environment interaction in the genetic evaluation of dairy cattle reduces accuracy but may increase selection intensity
}

\author{
Margot Slagboom, ${ }^{1 *} \odot$ A. Christian Sørensen, ${ }^{2}$ Jørn Rind Thomasen, ${ }^{3} \odot$ Huiming Liu, ${ }^{1}$ Morten Kargo, ${ }^{1}$ \\ and Line Hjortø ${ }^{2}$ \\ ${ }^{1}$ Centre for Quantitative Genetics and Genomics, Aarhus University, Blichers Allé, 8830 Tjele, Denmark \\ ${ }^{2}$ SEGES, Agro Food Park 15, 8200 Aarhus N, Denmark \\ ${ }^{3}$ VikingGenetics, Ebeltoftvej 16, 8960 Randers S $\varnothing$, Denmark
}

\begin{abstract}
Genotype by environment interaction $(\mathrm{G} \times \mathrm{E})$ may exist for traits that are expressed in different environments. The $\mathrm{G} \times \mathrm{E}$ is often ignored in the genetic evaluation of selection candidates. We hypothesized that genetic gain in 2 environments is always higher when the true value of the genetic correlation $\left(\mathrm{r}_{\mathrm{g}}\right)$ between traits expressed in different environments is considered in the genetic evaluation. We tested this hypothesis by stochastic simulation of dairy cattle breeding programs in a mainstream and a niche environment. The $r_{g}$ was varied from 0 to 1 in steps of 0.1 . We simulated the following 3 scenarios: 1Trait_1Index, 2Traits_1Index, and 2Traits_2Indices. The $\mathrm{G} \times \mathrm{E}$ was ignored in the genetic evaluation in the scenario with 1Trait and included in scenarios with 2Traits. Selection was based on the mainstream selection index in both environments in scenarios with 1Index. Selection in the mainstream environment was based on the mainstream selection index and selection in the niche environment was based on the niche selection index in the scenario with 2Indices. With moderate $\mathrm{G} \times \mathrm{E}$ ( $\mathrm{r}_{\mathrm{g}}$ between 0.6 and 0.9 ), the highest genetic gain was achieved in the niche environment by selecting for the mainstream selection index and ignoring $\mathrm{G} \times \mathrm{E}$. At lower $\mathrm{r}_{\mathrm{g}}$, the highest genetic gain was achieved when considering $\mathrm{G} \times \mathrm{E}$ and selecting for the niche selection index. For the mainstream environment, it was never an advantage to ignore $\mathrm{G} \times \mathrm{E}$. Therefore, although our hypothesis was confirmed in most cases, there were cases where ignoring $\mathrm{G} \times \mathrm{E}$ was the better option, and using the correct evaluation led to inferior genetic gain. The results of the current study can be used in animal breeding programs that encompass multiple environments.
\end{abstract}

Received June 15, 2021.

Accepted July 27, 2021.

*Corresponding author: margotslagboom@qgg.au.dk
Key words: genotype by environment interaction, breeding program, genetic evaluation, dairy cattle

\section{INTRODUCTION}

Genotype by environment interaction $(\mathbf{G} \times \mathbf{E})$ may exist for traits that are expressed in different environments. This causes the genetic correlation $\left(\mathbf{r}_{\mathrm{g}}\right)$ for a trait that is measured in different environments to be $<1$. For example, the $r_{g}$ between milk production measured in the Nordic countries and female fertility measured in New Zealand is 0.60 (International Bull Evaluation Service, 2020a), and for milk production the $r_{g}$ is 0.69 (International Bull Evaluation Service, 2020b). Genotype by environment interaction may also exist within one country when traits are expressed in different environments such as organic and conventional dairy production (Nauta et al., 2006; Sundberg et al., 2010; Liu et al., 2019). This is of importance in animal breeding programs where multiple environments are included. The reason for this is that breeding for a trait in one environment might result in a suboptimal result for the same trait in another environment because $\mathrm{G} \times \mathrm{E}$ can cause re-ranking of breeding bulls.

With the implementation of genomic selection, there is another important aspect that is affected by $\mathrm{G} \times \mathrm{E}$ : information on the association between genotypes and phenotypes from animals in a reference population. With $r_{g}$ of 1 , this association can be efficiently used across environments, but when $r_{g}$ decreases, so does the value of information from another environment. Slagboom et al. (2019) showed that the association between genotypes and phenotypes used across environments can still increase genetic gain when $r_{g}$ was as low as 0.4 , but this increase was a lot higher at high $\mathrm{r}_{\mathrm{g}}$. Thus, $\mathrm{G} \times \mathrm{E}$ has an effect on the value of information on a trait that is measured in different environments.

In practice, $\mathrm{G} \times \mathrm{E}$ is often ignored in the genetic evaluation of selection candidates, and traits are assumed to be caused by the same genes in different environments 
Table 1. Overview of simulated scenarios ${ }^{1}$

\begin{tabular}{llll}
\hline & $\begin{array}{l}\mathrm{G} \times \mathrm{E} \text { in } \\
\text { genetic } \\
\text { evaluation }\end{array}$ & $\begin{array}{l}\text { Selection index } \\
\text { in mainstream } \\
\text { environment }\end{array}$ & $\begin{array}{l}\text { Selection index } \\
\text { in niche } \\
\text { environment }\end{array}$ \\
\hline $\begin{array}{l}\text { 1Trait_1Index } \\
\text { 2Traits_1Index }\end{array}$ & No & Mainstream & Mainstream \\
2Traits_2Indices & Yes & Mainstream & Mainstream \\
\hline
\end{tabular}

${ }^{1}$ 1Trait_1Index: genotype by environment interaction $(\mathrm{G} \times \mathrm{E})$ was ignored in the genetic evaluation and selection was based on the mainstream selection index in both environments. 2Traits_1Index: $\mathrm{G} \times \mathrm{E}$ was included in the genetic evaluation and selection was based on the mainstream selection index in both environments. 2Traits_2Indices: $\mathrm{G} \times \mathrm{E}$ was included in the genetic evaluation. Selection in the mainstream environment was based on the mainstream selection index and selection in the niche environment was based on the niche selection index.

(e.g., $r_{g}$ is assumed to be 1 ). The consequence is a single breeding program across different environments or production systems allowing a high selection intensity, which can result in high genetic gain (Mulder et al., 2006). The potential cost of assuming $r_{g}$ to be 1 is a lower accuracy of selection if the true $r_{\mathrm{g}}$ differs from 1 . Therefore, suboptimal selection decisions will be made that affect the genetic gain generated in the breeding programs.

The objective of this research was to study genetic gain when ignoring $\mathrm{G} \times \mathrm{E}$ in the genetic evaluation of dairy cattle. We hypothesized that the annual true genetic gain in 2 environments is always higher when the true value of $r_{g}$ is considered in the genetic evaluation. We tested this hypothesis by stochastic simulation of dairy cattle breeding programs with 2 environments: a mainstream and a niche environment. We varied the levels of $\mathrm{G} \times \mathrm{E}$ interactions and the selection index; selection decisions were based on the index in the mainstream environment or the niche selection index.

\section{MATERIALS AND METHODS}

\section{Design}

In this study, we simulated 3 scenarios with 2 environments: one niche and one mainstream dairy production environment. The mainstream environment emulated a conventional dairy production environment in a Northern European country, and the niche environment emulated a niche production environment such as organic dairy production. Cattle breed and country were assumed to be the same for the 2 environments. In each environment, we simulated one trait with a heritability of 0.20 . This one trait can be interpreted as a total merit index for each environment. The $r_{g}$ between the trait in each environment was used as a measure of $\mathrm{G} \times \mathrm{E}$ interaction. The $\mathrm{r}_{\mathrm{g}}$ ranged from 0 to 1 at intervals of 0.1 . The 3 scenarios were simulated for this range of $r_{g}$. Scenarios differed in the inclusion of one or 2 traits in the genetic evaluation, which meant that $\mathrm{G} \times \mathrm{E}$ was either ignored (one trait) or included (2 traits). Both environments were simulated simultaneously in each scenario. Selection indices also differed among scenarios (Table 1):

(1) 1Trait_1Index: $\mathrm{G} \times \mathrm{E}$ was ignored in the genetic evaluation, and selection was based on the mainstream selection index in both environments.

(2) 2Traits_1Index: $\mathrm{G} \times \mathrm{E}$ was included in the genetic evaluation and selection was based on the mainstream selection index in both environments.

(3) 2Traits_2Indices: $\mathrm{G} \times \mathrm{E}$ was included in the genetic evaluation. Selection in the mainstream environment was based on the mainstream selection index and selection in the niche environment was based on the niche selection index.

Scenario 1Trait_2Indices would be a logical fourth scenario in a 2-by-2 design. However, there cannot be 2 indices when only a single trait is genetically evaluated.

\section{Simulated Population}

A base population of unrelated animals was sampled and distributed among environments and among the age classes 0 to 5 for females and 0 to 1 for males. Each environment had a breeding nucleus that resembled the breeding nucleus of a dairy cattle population, each nucleus consisting of 10,000 lactating cows (Figure 1). Each year and within each environment, 1,500 oneyear-old heifers were selected for genotyping based on parent average. The 225 best genotyped heifers were selected based on genomically enhanced breeding values (GEBV) within each environment and bred by multiple ovulation and embryo transfer. Selected heifers produced 3 offspring from 2 different bulls, thus 6 offspring in total per heifer. The remaining females produced one viable offspring each. Each offspring had equal probabilities of being male or female. 


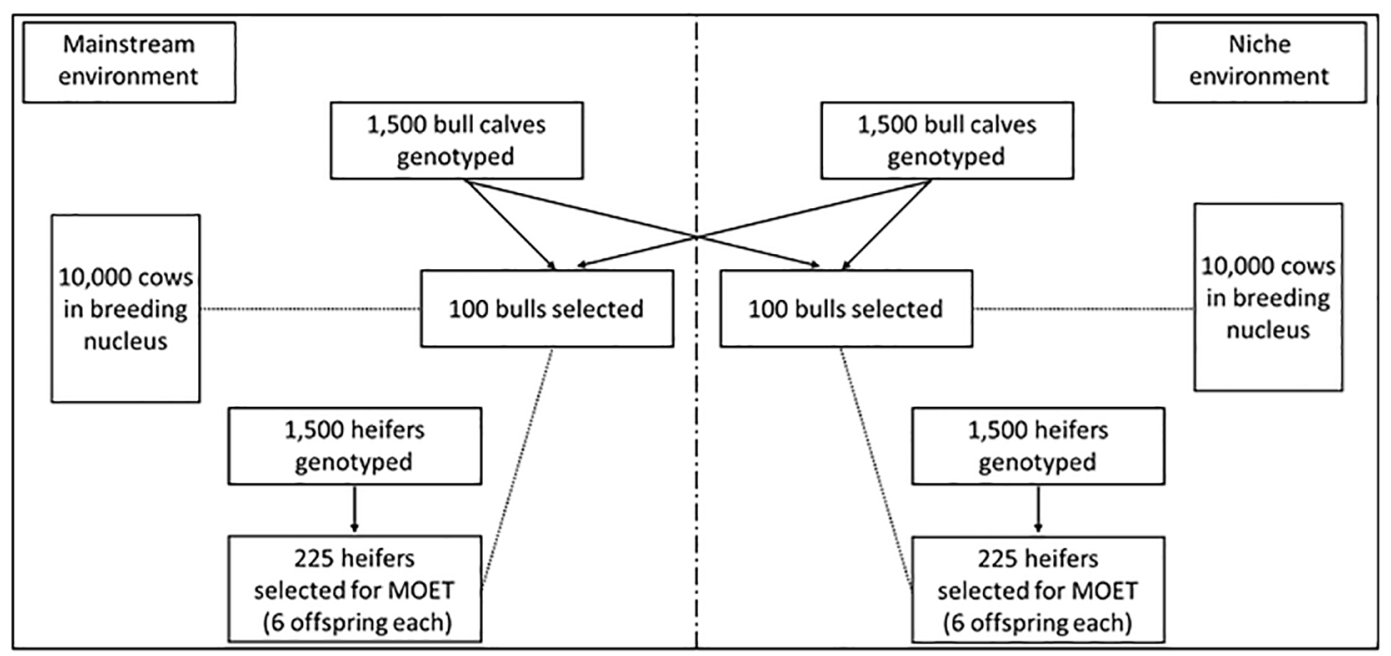

Figure 1. Schematic overview of the breeding scheme used in this study. Dotted lines indicate that groups of animals were mated to each other and arrows indicate that a proportion of animals were selected. Selection of bulls from the foreign environment was possible. MOET $=$ multiple ovulation and embryo transfer.

Each year and within each environment, 1,500 oneyear-old bulls were selected for genotyping based on parent average. All one-year-old bulls that were born as a result of multiple ovulation and embryo transfer were included in this genotyping step, regardless of their parent average. The 100 best genotyped bulls were selected for AI across environments based on GEBV and were used equally among all females. Bulls were selected in 2 selection steps, one for each group of cows (niche or mainstream). In scenario 2Traits_2Indices, the 2 selection steps were based on different selection indices, whereas in the other 2 scenarios the selection indices were the same in the 2 environments. This means that different more or less overlapping sets of bulls could be selected to be bred to the niche cows and the mainstream cows in scenario 2Traits_2Indices.

All unselected candidates as well as all females older than $5 \mathrm{yr}$, and all bulls older than one year were culled. In addition, $15 \%$ of all animals between one and $5 \mathrm{yr}$ were randomly culled before selection decisions were made to reflect involuntary culling. Each scenario was run for 30 years with overlapping generations and was replicated 100 times. We used the stochastic simulation program ADAM to run the simulations (Pedersen et al., 2009).

True breeding values for all traits were sampled for all animals. Within each environment, phenotypic values were sampled for all cows when they finished first lactation. Genomic information was modeled using pseudogenomic selection; we did not simulate chromosomes, genes, or markers, but instead direct genomic values (DGV) for the trait in both environments were sampled for all genotyped animals. This method is similar to the method developed by Dekkers (2007) as implemented by Buch et al. (2012). We simulated complete exchange of genomic and phenotypic information across environments.

\section{Genetic Evaluation}

The GEBV were predicted using a multivariate best linear unbiased prediction model. Candidates were selected by truncation and parents were mated randomly in all generations. We used the DMU package to predict GEBV (Madsen and Jensen, 2013). Genetic variances were set to 1 , and genetic gain was expressed in genetic standard deviation $\left(\sigma_{A}\right)$ units. The genetic and environmental (co)variance matrices, $\mathbf{G}$ and $\mathbf{R}$, were used to simulate true breeding values, phenotypic values, and DGV:

$$
\begin{aligned}
& \mathbf{G}= \\
& {\left[\begin{array}{cccc}
1 & r_{g} & 0.707 & 0.707 \times r_{g} \\
r_{g} & 1 & 0.707 \times r_{g} & 0.707 \\
0.707 & 0.707 \times r_{g} & 1 & 0.707 \times 0.707 \times r_{g} \\
0.707 \times r_{g} & 0.707 & 0.707 \times 0.707 \times r_{g} & 1
\end{array}\right],} \\
& \mathbf{R}=\left[\begin{array}{cccc}
\frac{1-h^{2}}{h^{2}}=4 & 0 & 0 & 0 \\
0 & 4 & 0 & 0 \\
0 & 0 & 0.01 & 0 \\
0 & 0 & 0 & 0.01
\end{array}\right],
\end{aligned}
$$


where $r_{g}$ is the genetic correlation between the trait in each environment, representing $\mathrm{G} \times \mathrm{E}$, and $h^{2}$ is the heritability. The first 2 traits in $\mathbf{G}$ and $\mathbf{R}$ are the observed traits in environments 1 and 2 and the last 2 traits are the DGV in environments 1 and 2. The DGV had a genetic correlation to the observed trait of 0.707 , which implies a reliability of 0.50 . The heritability of the DGV is the repeatability of the marker information and is approximately equal to 1 . The matrix $\mathbf{G}$ was not positive definite when $r_{g}$ was equal to 1 and therefore this matrix was bent by means of the method proposed by Hayes and Hill (1981).

\section{Statistical Analysis}

Annual genetic gain in $\sigma_{A}$ units was calculated by regressing true genetic levels of the observed traits in the last $10 \mathrm{yr}$ of the simulation on year, for each replicate and then averaged across replicates. Genetic gain per year was compared between scenarios for each environment separately for the range of $r_{g}$ from 0 to 1 . All statistical analyses were performed in $\mathrm{R}$ statistical software version 4.0.2 (https://r-project.org).

Relative genetic gain was calculated in scenario 2Traits_2Indices and 2Traits_1Index compared with genetic gain in scenario 1Trait_1Index using Equation [1]:

$$
G_{r e l}=\frac{\Delta G_{\text {alt }, i}}{\Delta G_{1 \text { Trait_1Index }}} \times 100 \%,
$$

where $G_{r e l}=$ relative genetic gain in percentages, $\Delta G_{a l t, i}$ $=$ genetic gain per year in alternative scenario $i(i=$ 2Traits_2Indices, 2Traits_1Index), and $\Delta G_{1 \text { Trait_1Index }}$

$=$ genetic gain per year in scenario 1Trait_1Index. Relative genetic gain was calculated within each environment.

The percentage of bulls selected each year for breeding in one environment but originating from the foreign environment was calculated using Equation [2]:

$$
P_{\text {foreign }}=\frac{N_{\text {foreign }}}{N_{\text {foreign }}+N_{\text {home }}} \times 100 \% \text {, }
$$

where $P_{\text {foreign }}=$ percentage of selected breeding bulls that originate from the foreign environment, $N_{\text {foreign }}$ $=$ number of selected breeding bulls that originate from the foreign environment, and $N_{\text {home }}=$ number of selected breeding bulls that originate from the home environment.
The selection intensity was first calculated per breeding program and environment of origin using the proportion of selected bulls. Then the realized selection intensity per breeding program was calculated using a weighted average of the selection intensity per breeding program and environment of origin weighted by the proportion of bulls selected within each environment of origin. The accuracy of selection was calculated as the correlation between true and estimated breeding values $\left(\mathbf{r}_{\mathrm{IH}}\right)$ from the output of the simulation. The regression of true breeding values on estimated breeding values was calculated to assess potential bias in the estimated breeding values. The rate of inbreeding per generation was calculated for each replicate as 1 minus the exponential of the regression coefficient of the natural logarithm of 1 minus mean inbreeding on the average generation equivalent for each year (Falconer and Mackay, 1996). The presented rate of inbreeding per generation was then obtained by averaging over replicates for each scenario.

\section{RESULTS}

Annual genetic gain in $\sigma_{A}$ units was equal for all 3 scenarios and both environments at $\mathrm{r}_{\mathrm{g}}$ of 1 (Figure 2 and Figure 3). In scenario 1Trait_1Index the 2 environments showed similar trends; genetic gain steadily increased with increasing $\mathrm{r}_{\mathrm{g}}$. Scenario 2Traits_2Indices also showed similar trends in the 2 environments; at genetic correlations below 0.7 , genetic gain was independent of the genetic correlation. At genetic correlations above 0.7 , genetic gain increased with increasing genetic correlation. Scenario 2Traits_1Index resulted in a slight increase at high $r_{g}$ in the mainstream environment, whereas this scenario resulted in no genetic gain at $r_{g}$ of 0 and a large increase with increasing $r_{g}$ in the niche environment. The most beneficial scenario in terms of genetic gain for the mainstream environment was to include $\mathrm{G} \times \mathrm{E}$ and breed for the mainstream index in both environments (2Traits_1Index); genetic gain was up to $40 \%$ higher compared with genetic gain in scenario 1Trait_1Index (at $r_{\mathrm{g}}=0$, results not shown). The difference in genetic gain between these 2 scenarios decreased as $r_{g}$ increased. For the niche environment, the most beneficial scenario was dependent on $r_{g}$. At $r_{g}$ less than 0.6, scenario 2Traits_2Indices was the most beneficial, but if $r_{g}$ was in the range of 0.6 to 0.9, scenario 1Trait_1Index yielded the highest genetic gain by as much as $4 \%$. Thus, ignoring $\mathrm{G} \times \mathrm{E}$ increased genetic gain for this range of $r_{g}$.

Scenario 1Trait_1Index resulted in a selected proportion of bulls from each environment of about 50\% (Figure 4 and 5). In scenario 2Traits_1Index more 


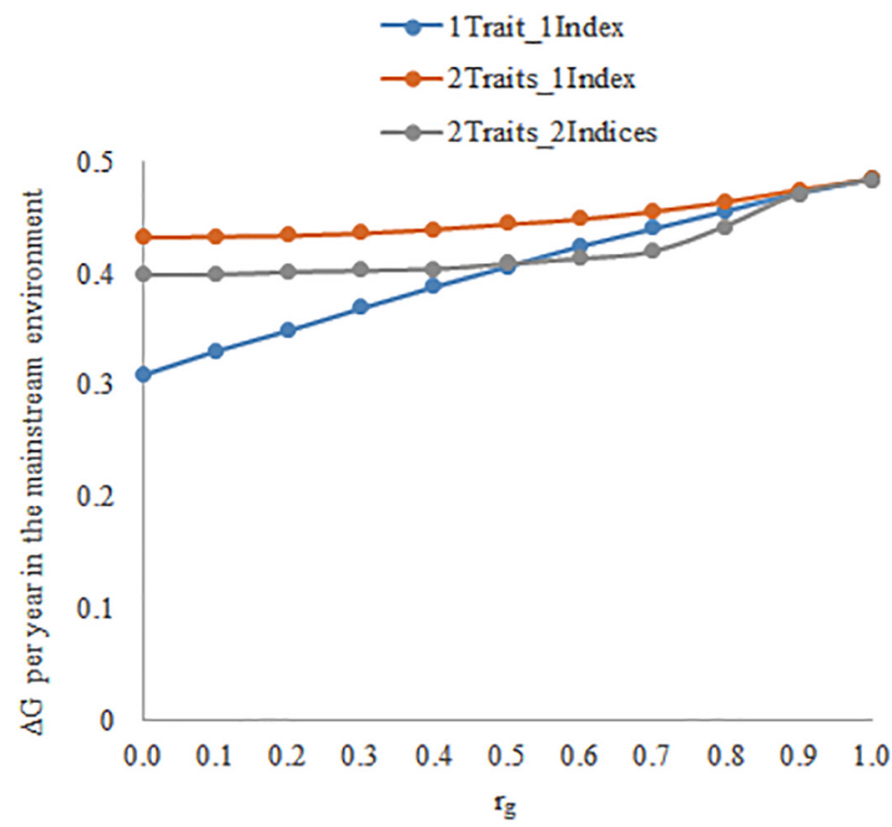

Figure 2. Annual genetic gain $(\Delta \mathrm{G})$ in the mainstream environment at different correlations $\left(\mathrm{r}_{\mathrm{g}}\right)$ between the trait in each environment for all 3 scenarios. 1Trait_1Index: genotype by environment interaction $(\mathrm{G} \times \mathrm{E})$ was ignored in the genetic evaluation and selection was based on the mainstream selection index in both environments. 2Traits_1Index: $\mathrm{G} \times \mathrm{E}$ was included in the genetic evaluation and selection was based on the mainstream selection index in both environments. 2Traits_2Indices: $\mathrm{G} \times \mathrm{E}$ was included in the genetic evaluation. Selection in the mainstream environment was based on the mainstream selection index and selection in the niche environment was based on the niche selection index.

than $50 \%$ of breeding bulls originated from the mainstream environment. In scenario 2Traits_2Indices no bulls were selected from the foreign environment until $r_{g}$ was approximately or above 0.7 . The realized selection intensity was 2.23 in scenarios 1Trait_1Index and 2Traits_1Index regardless of environment and $r_{g}$. In scenario 2Traits_2Indices the realized selection intensity was as low as 1.94 when $r_{g}$ was below or equal to 0.6 , above which the selection intensity slowly increased to 2.23 at $r_{\mathrm{g}}$ of 1 (results not shown).

Bulls born in the niche environment in scenario 2Trait_2Indices had the highest correlation between true breeding values and the mainstream selection index regardless of $r_{g}$ (Figure 6), whereas bulls born in the mainstream environment in scenario 2Trait_2Indices had the highest correlation between true breeding values and the niche selection index regardless of $r_{g}$ (Figure 7). Ignoring $\mathrm{G} \times \mathrm{E}$ greatly decreased $\mathrm{r}_{\mathrm{IH}}$ at low $\mathrm{r}_{\mathrm{g}}$ for both selection indices (scenario 1Trait_1Index). An even more extreme decrease was seen in scenario 2Traits_1Index for the niche selection index (Figure 7). The regression of true breeding values on estimated breeding values was very close to unity in scenarios
2Traits_1Index and 2Traits_2Indices independent of the genetic correlation (results not shown). In scenario 1Trait_1Index the regression had a value of approximately 0.75 at $r_{g}$ of 0 and increased toward unity with increasing $r_{g}$ (results not shown). The rate of inbreeding was approximately $0.1 \%$ per generation for all scenarios, with slightly higher rates at lower $r_{g}$ when including $\mathrm{G} \times \mathrm{E}$ (results not shown).

\section{DISCUSSION}

In this study, several scenarios were simulated that differed in the inclusion of $\mathrm{G} \times \mathrm{E}$ in the genetic evaluation and the use of one selection index for 2 environments or the use of 2 environment-specific selection indices. The results did not allow us to confirm our hypothesis that the annual true genetic gain in 2 environments is always higher when the true value of $r_{\mathrm{g}}$ is considered in the genetic evaluation. In cases of a genetic correlation between the simulated environments in the range of 0.6 to 0.9 , farmers in the niche environment were better off by cooperating with the mainstream environment while ignoring $\mathrm{G} \times \mathrm{E}$ compared with including $\mathrm{G} \times \mathrm{E}$ and

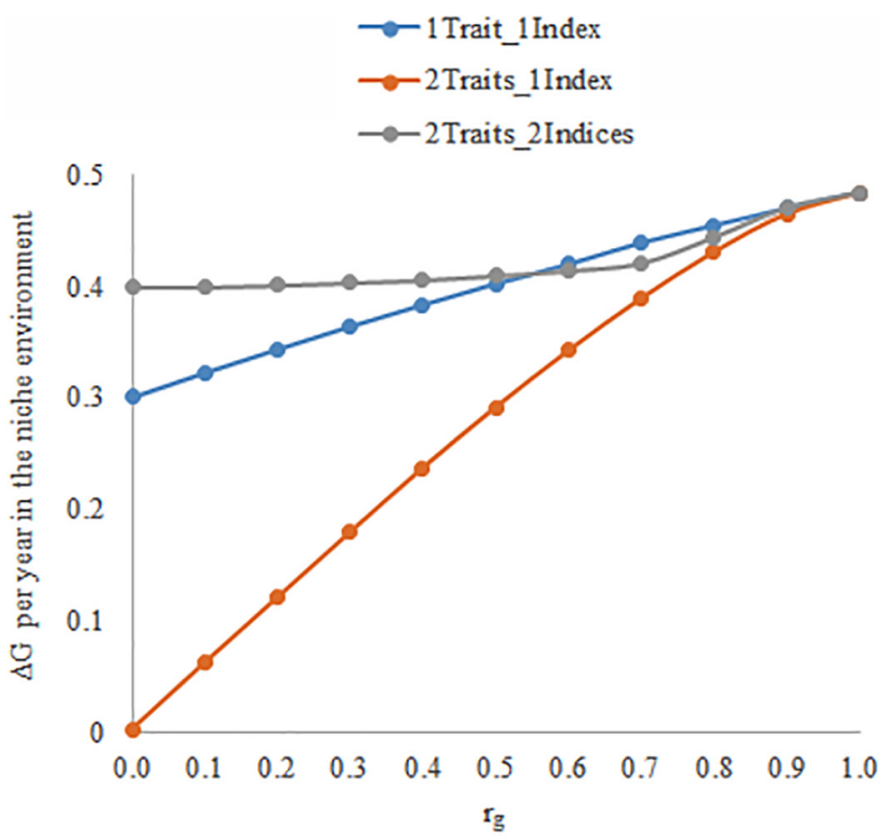

Figure 3. Annual genetic gain $(\Delta \mathrm{G})$ in the niche environment at different correlations $\left(\mathrm{r}_{\mathrm{g}}\right)$ between the trait in each environment for all 3 scenarios. 1Trait_1Index: genotype by environment interaction $(\mathrm{G} \times \mathrm{E})$ was ignored in the genetic evaluation and selection was based on the mainstream selection index in both environments. 2Traits_1Index: $\mathrm{G} \times \mathrm{E}$ was included in the genetic evaluation and selection was based on the mainstream selection index in both environments. 2Traits_2Indices: $\mathrm{G} \times \mathrm{E}$ was included in the genetic evaluation. Selection in the mainstream environment was based on the mainstream selection index and selection in the niche environment was based on the niche selection index. 
using their own selection index even with exchange of information and germ plasm. The best case for farmers in the mainstream environment was independent of the genetic correlation; the highest genetic gain was achieved by taking account of $\mathrm{G} \times \mathrm{E}$ but using the mainstream selection index for all selection decisions. This was, however, always the worst case for farmers in the niche environment. Therefore, although our hypothesis was confirmed in most cases, there were cases where ignoring $\mathrm{G} \times \mathrm{E}$ was the better option and using the correct evaluation led to inferior genetic gain.

\section{Trade-Off Between Selection Intensity and $r_{I H}$}

The most interesting and unexpected result of this study is seen in the niche environment when $r_{g}$ was 0.6 to 0.9 (Figure 3). In this range of $r_{g}$, scenario 1Trait_1Index resulted in higher genetic gain compared with genetic gain in scenario 2Traits_2Indices. The driving forces behind genetic gain in the different scenarios in this study were the selection intensity and $\mathrm{r}_{\mathrm{IH}}$. The selection intensity was low in scenario

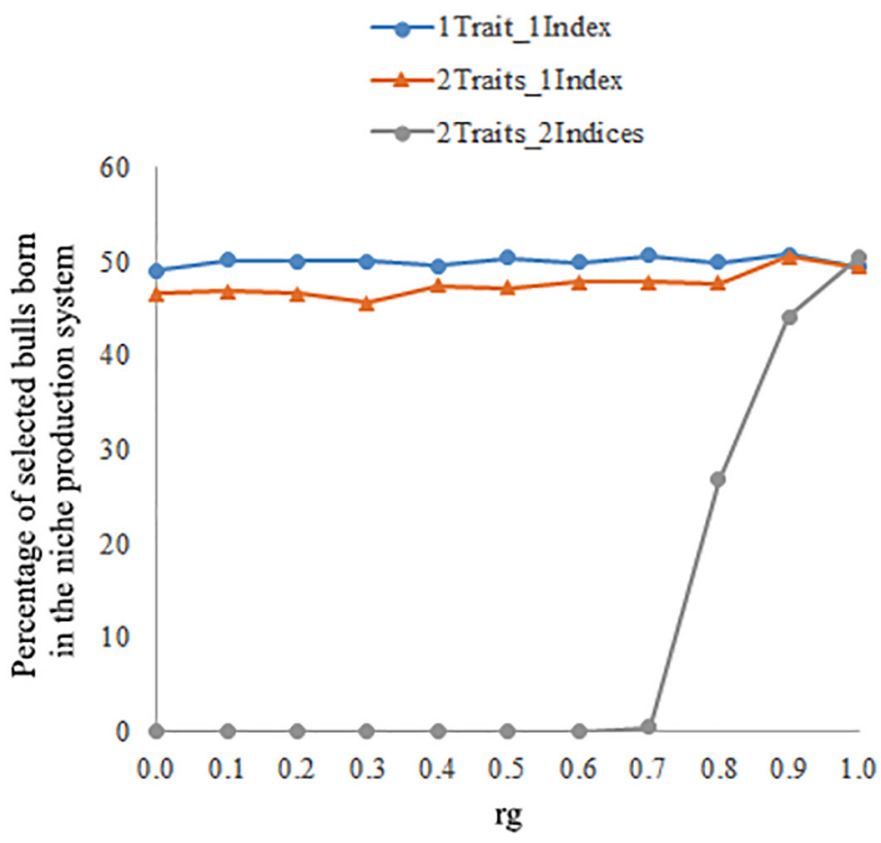

Figure 4. Proportion of selected bulls in the mainstream environment born in the niche environment in generation 30 for all 3 scenarios. 1Trait_1Index: genotype by environment interaction $(\mathrm{G} \times \mathrm{E})$ was ignored in the genetic evaluation and selection was based on the mainstream selection index in both environments. 2Traits_1Index: $\mathrm{G} \times \mathrm{E}$ was included in the genetic evaluation and selection was based on the mainstream selection index in both environments. 2Traits_2Indices: $\mathrm{G} \times \mathrm{E}$ was included in the genetic evaluation. Selection in the mainstream environment was based on the mainstream selection index and selection in the niche environment was based on the niche selection index.

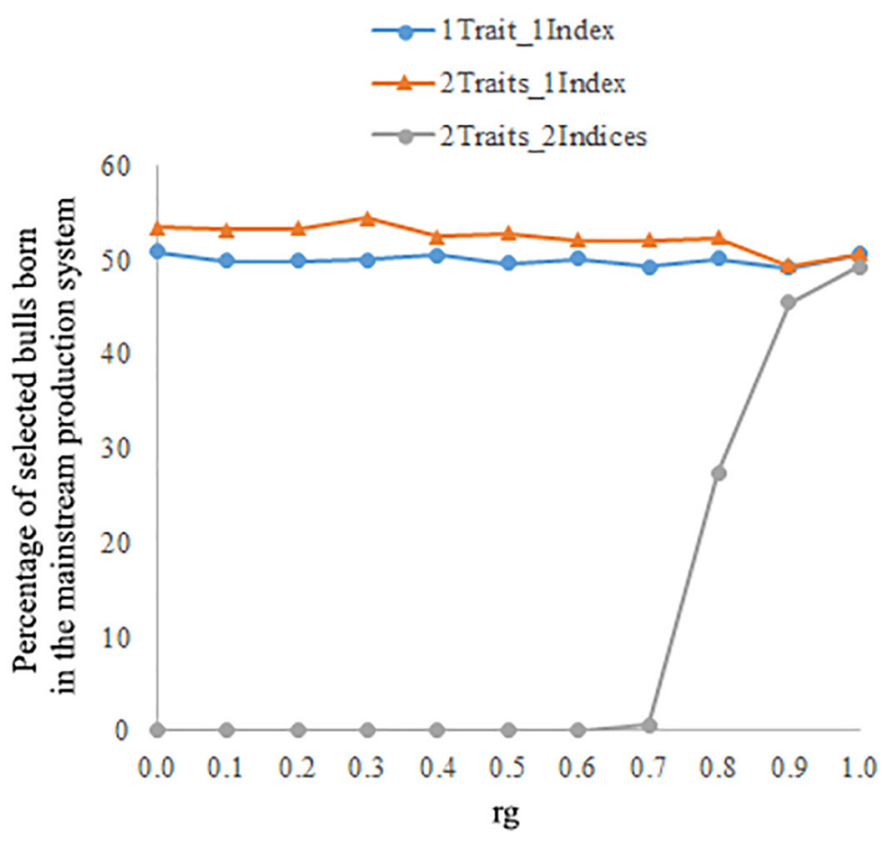

Figure 5. Proportion of selected bulls in the niche environment born in the mainstream environment in generation 30 for all 3 scenarios. 1Trait_1Index: genotype by environment interaction $(\mathrm{G} \times \mathrm{E})$ was ignored in the genetic evaluation and selection was based on the mainstream selection index in both environments. 2Traits_1Index: $\mathrm{G} \times \mathrm{E}$ was included in the genetic evaluation and selection was based on the mainstream selection index in both environments. 2Traits_2Indices: $\mathrm{G} \times \mathrm{E}$ was included in the genetic evaluation. Selection in the mainstream environment was based on the mainstream selection index and selection in the niche environment was based on the niche selection index.

2Traits_2Indices until bulls started being selected from the foreign environment at $r_{g}=0.7$ (Figure 5). This specific $r_{g}$ is also known as the split-point correlation, the point where selection indices in different environments become so similar that it is more beneficial for genetic gain to select bulls from the foreign environment (Mulder and Bijma, 2006; Slagboom et al., 2019). The selection intensity and consequently genetic gain increases by including selection candidates from both environments. The selection intensity was high regardless of $r_{g}$ in scenario 1Trait_1Index, but $r_{I H}$ was lower in this scenario because $\mathrm{G} \times \mathrm{E}$ was ignored. The effect of the high selection intensity from breeding for the same index in the 2 environments was more important than the effect of the increased $r_{I H}$ when taking $\mathrm{G} \times \mathrm{E}$ into account when $r_{g}$ was 0.6 to 0.9 . Thus, contradictory to what we hypothesized, it was better to ignore $\mathrm{G} \times \mathrm{E}$ for this range of $r_{g}$.

\section{Genetic Gain in the Different Scenarios}

Genetic gain in scenario 1Trait_1Index was the same for both environments because the 2 environ- 
ments were treated as one. Hence, the 2 environments did not diverge. Scenario 2Traits_1Index resulted in the highest genetic gain regardless of $r_{g}$ in the mainstream environment, whereas this scenario resulted in the lowest annual genetic gain regardless of $r_{g}$ in the niche environment. The reason for this is that all phenotypes were used according to their true information content with respect to the mainstream selection index and the 2 environments were not allowed to diverge. Consequently, in the niche environment the genetic gain decreased to 0 as $r_{g}$ decreased to 0 . In contrast, in scenario 2Traits_2Indices, the 2 environments were treated separately and were allowed to diverge. The inclusion of $\mathrm{G} \times \mathrm{E}$ in the genetic evaluation and breed-

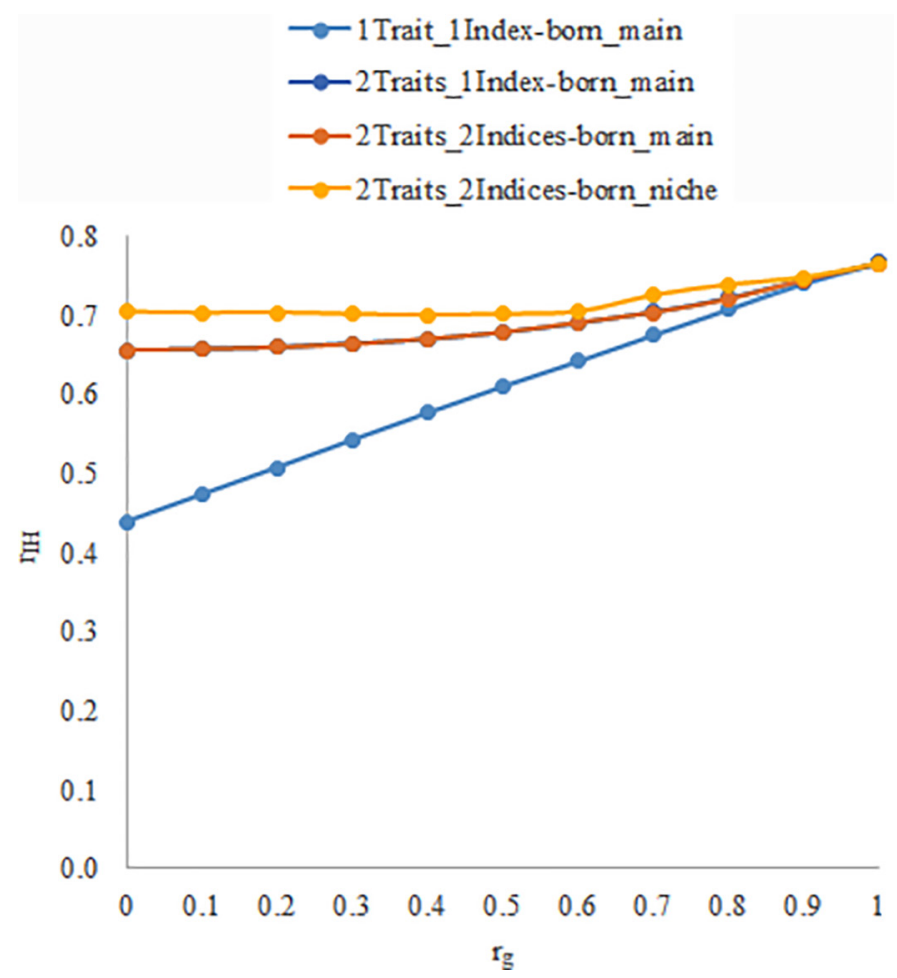

Figure 6. Correlations between true breeding values and the selection index for the mainstream environment $\left(\mathrm{r}_{\mathrm{IH}}\right)$ of male candidates for selection born in either the mainstream environment or the niche environment, at different correlations $\left(\mathrm{r}_{\mathrm{o}}\right)$ between the trait in each environment for all 3 scenarios in generations 21 to 30. 1Trait_1Index: genotype by environment interaction $(\mathrm{G} \times \mathrm{E})$ was ignored in the genetic evaluation and selection was based on the mainstream selection index in both environments. 2Traits_1Index: $\mathrm{G} \times \mathrm{E}$ was included in the genetic evaluation and selection was based on the mainstream selection index in both environments. 2Traits_2Indices: $\mathrm{G} \times \mathrm{E}$ was included in the genetic evaluation. Selection in the mainstream environment was based on the mainstream selection index and selection in the niche environment was based on the niche selection index. For the scenarios with 1 index, only bulls that were born in the mainstream environment are included because $r_{I H}$ was the same for bulls born in both environments. $\mathrm{r}_{\mathrm{IH}}$ of 2Traits_1Index-born_main is not visible because 2Traits_1Index-born_main and 2Traits_2Indices-born_main resulted in equal $\mathrm{r}_{\mathrm{IH}}$.

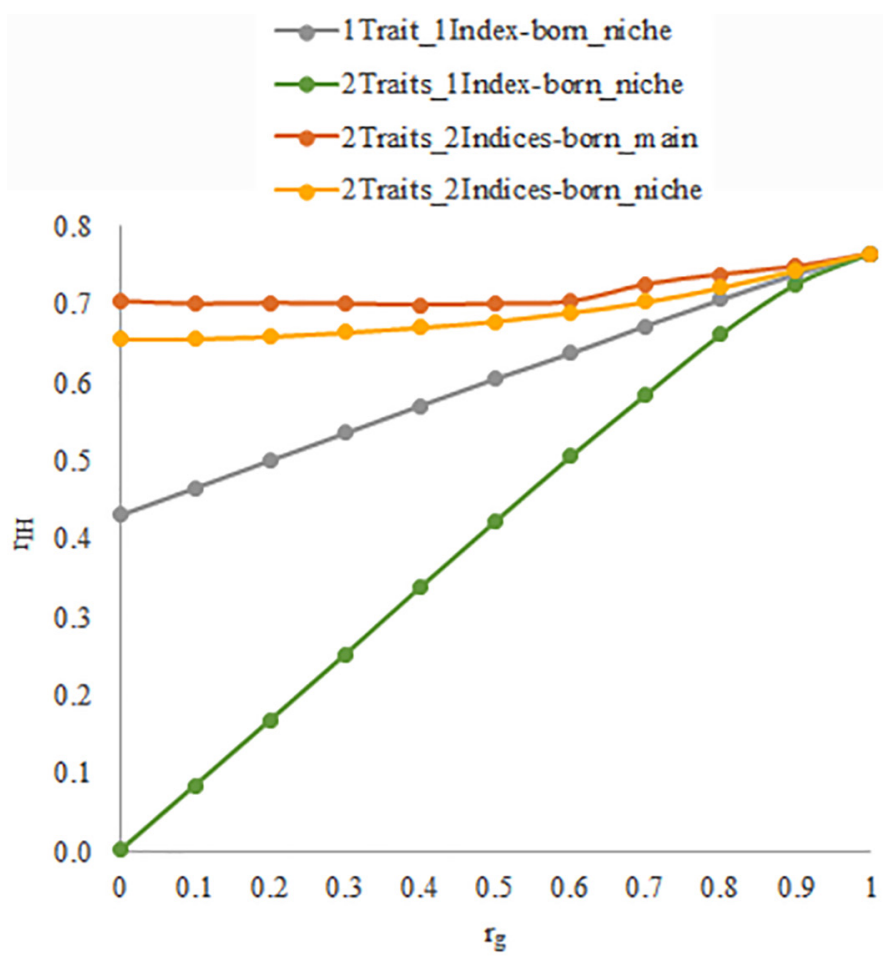

Figure 7. Correlations between true breeding values and the selection index for the niche environment $\left(\mathrm{r}_{\mathrm{IH}}\right)$ of male candidates for selection born in either the mainstream environment or the niche environment, at different correlations $\left(\mathrm{r}_{\mathrm{g}}\right)$ between the trait in each environment for all 3 scenarios in generations 21 to 30. 1Trait_1Index: genotype by interaction $(\mathrm{G} \times \mathrm{E})$ was ignored in the genetic evaluation and selection was based on the mainstream selection index in both environments. 2Traits_1Index: $\mathrm{G} \times \mathrm{E}$ was included in the genetic evaluation and selection was based on the mainstream selection index in both environments. 2Traits_2Indices: $\mathrm{G} \times \mathrm{E}$ was included in the genetic evaluation. Selection in the mainstream environment was based on the mainstream selection index and selection in the niche environment was based on the niche selection index. For the scenarios with 1 index, only bulls that were born in the niche environment are included because $\mathrm{r}_{\mathrm{IH}}$ was the same for bulls born in both environments.

ing for different indices greatly influenced genetic gain in the 2 environments.

The selection intensity was constant and high (2.23) in the scenarios with one selection index. All breeding bulls were selected in one selection step and therefore the exact same group of bulls were bred to both the niche and the mainstream cow breeding nucleus. Ignoring or including $\mathrm{G} \times \mathrm{E}$ in these scenarios did not change the selection intensity. When the selection index was changed so that both environments bred for the trait in their own environment and $\mathrm{G} \times \mathrm{E}$ was included in the genetic evaluation (scenario 2Traits_2Indices), different bulls could be selected to be bred to the niche and to the mainstream cows. Therefore, the total selected proportion of breeding bulls was higher compared with the other scenarios. This difference was only seen at low $r_{g}$, at high $r_{g}$ the selected proportion was the same because 
the selection indices were very similar. This is reflected in the difference in selection intensity between scenarios and at different $r_{g}$; the selection intensity decreased from 2.23 at an $\mathrm{r}_{\mathrm{g}}$ of 0.9 to a selection intensity of 1.94 at an $r_{g}$ of 0.6 and lower in scenario 2Traits_2Indices. Hence, $\mathrm{G} \times \mathrm{E}$ only influenced the selection intensity if environments bred for an environment-specific selection index.

The correlation between true and estimated breeding values decreased when $r_{g}$ decreased and $G \times E$ was ignored in the genetic evaluation, because phenotypes from the niche environment were wrongfully labeled as the right phenotypes for the mainstream selection index (scenario 1Trait_1Index in Figure 6 and 7). This resulted in biased estimated breeding values; the regression of true breeding values on estimated breeding values decreased from unity toward approximately 0.75 at $\mathrm{r}_{\mathrm{g}}$ of 0 (results not shown). Including $\mathrm{G} \times \mathrm{E}$ in the genetic evaluation increased $r_{I H}$ for the mainstream selection index because phenotypic information from the 2 environments were used appropriately resulting in no bias (scenario 2Traits_1Index in Figure 6). Including $\mathrm{G} \times \mathrm{E}$ in the genetic evaluation decreased $\mathrm{r}_{\mathrm{IH}}$ for the niche selection index to 0 at $r_{g}$ of 0 when selection was aimed at improving the mainstream trait (scenario 2Traits_1Index in Figure 7). The reason for this is that breeding values were estimated for the mainstream selection index and phenotypes of the animals in the niche environment were rightfully labeled as a correlated phenotype for that index. In scenario 2Traits_2Indices the niche environment shifted to an environment-specific index, which greatly increased $\mathrm{r}_{\mathrm{IH}}$. Bulls born in the foreign environment had higher $r_{I H}$ than bulls born in the home environment likely because there was less reduction in variance of the home selection index due to the Bulmer effect (Bulmer, 1971), which was not the trait under selection in the foreign environment. Therefore, although properly accounting for $\mathrm{G} \times \mathrm{E}$ in the genetic evaluation always maximizes the correlation between true and EBV, as expected by theory, the maximized accuracy is not always translated into maximized genetic gain.

\section{Simulation Input}

Input to the simulations in this study were based on parameters for Nordic Holstein. The one trait that was included had a heritability of 0.20 . A lower or higher heritability will decrease or increase genetic progress in each of the environments, but we do not expect the comparison between the different scenarios to be different. Similarly, in Mulder and Bijma (2006), estimates of the split-point correlation hardly differed with different heritabilities.
Genetic gain in this study was measured for one trait only. In reality, breeding goals encompass multiple traits. The inclusion of more traits may have changed the results of this study, but may have also made results harder to interpret. However, we believe that the results are still credible considering that the trait represents a total merit breeding goal.

\section{Implications}

The results of the current study can be used in animal breeding programs that encompass multiple environments but do not take account of $\mathrm{G} \times \mathrm{E}$. Genotype by environment interaction has been estimated in some countries between niche and mainstream production environments. For example, $r_{g}$ between organic and conventional dairy production environments within a country tend to be quite high (Liu et al., 2019; Nauta et al., 2006; Sundberg et al., 2010). In this case, higher genetic gain will be achieved with one breeding program for both environments that ignore genotype by environment interaction in the genetic evaluation of selection candidates. At lower $r_{g}$, for example between functional traits measured in the Nordic countries and New Zealand (International Bull Evaluation Service, 2014), more genetic progress will be achieved with separate indices that take $\mathrm{G} \times \mathrm{E}$ into account.

Even though genetic gain in the total merit index was higher when ignoring $\mathrm{G} \times \mathrm{E}$ for moderate $\mathrm{G} \times \mathrm{E}$ interactions, genetic gain on trait level may show a more desirable direction of genetic progress which cannot be shown by genetic gain in the total merit index alone. Slagboom et al. (2020) showed that a significant increase in genetic merit of functional traits can be achieved with a minor decrease in the aggregate genotype. This decrease in genetic gain might be acceptable if accompanied by a more desirable direction of selection on individual trait level.

\section{CONCLUSIONS}

The current study shows the effect of ignoring $\mathrm{G} \times \mathrm{E}$ in the genetic evaluation of dairy breeding programs. With moderate $\mathrm{G} \times \mathrm{E}$ ( $\mathrm{r}_{\mathrm{g}}$ between 0.6 and 0.9 ) it was better to ignore $\mathrm{G} \times \mathrm{E}$ in the genetic evaluation of the niche selection index because the higher selection intensity increased annual genetic gain more than the increase in correlation between true and estimated breeding values did. At lower $r_{g}$ between traits measured in different environments it was better for annual genetic gain to take $\mathrm{G} \times \mathrm{E}$ into account in the genetic evaluation. For the mainstream selection index, it was never an advantage to ignore $\mathrm{G} \times \mathrm{E}$. These results can 
be used in animal breeding programs that encompass multiple environments.

\section{ACKNOWLEDGMENTS}

The project $\mathrm{G} \times \mathrm{E}$ between organic and conventional systems was financially supported by the Danish Milk Levy Foundation and the Danish fund for Niche Farming. The authors have not stated any conflicts of interest.

\section{REFERENCES}

Buch, L. H., M. K. Sørensen, P. Berg, L. D. Pedersen, and A. C. Sørensen. 2012. Genomic selection strategies in dairy cattle: Strong positive interaction between use of genotypic information and intensive use of young bulls on genetic gain. J. Anim. Breed. Genet. 129:138-151. https://doi.org/10.1111/j.1439-0388.2011.00947.x

Bulmer, M. G. 1971. The effect of selection on genetic variability. Am. Nat. 105:201-211. https://doi.org/10.1086/282718.

Dekkers, J. C. M. 2007. Prediction of response to marker assisted and genomic selection using selection index theory. J. Anim. Breed. Genet. 124:331-341. https://doi.org/10.1111/j.1439-0388.2007 .00701.x.

Falconer, D. S., and T. F. C. Mackay. 1996. Introduction to Quantitative Genetics. 4th ed. Pearson.

Hayes, J. F., and W. G. Hill. 1981. Modification of estimates of parameters in the construction of genetic selection indices ('bending'). Biometrics 37:483-493. https://doi.org/10.2307/2530561.

International Bull Evaluation Service. 2014. Interbull routine genetic evaluation for female fertility traits, August 2014. https://interbull .org/web/static/mace_evaluations/1408r/fert1408r.pdf.

International Bull Evaluation Service. 2020a. Interbull routine genetic evaluation for female fertility traits, December 2020. https:// interbull.org/static/web/fertdoc2012r.pdf.

International Bull Evaluation Service. 2020b. Interbull routine genetic evaluation for production traits, December 2020. https://interbull .org/static/web/proddoc2012r.pdf.

Liu, A., G. Su, J. Höglund, Z. Zhang, J. Thomasen, I. Christiansen, Y. Wang, and M. Kargo. 2019. Genotype by environment interaction for female fertility traits under conventional and organic production systems in Danish Holsteins. J. Dairy Sci. 102:8134-8147. https://doi.org/10.3168/jds.2018-15482.

Madsen, P., and J. Jensen. 2013. A User's Guide to DMU. Version 6, release 5.2. Aarhus University.

Mulder, H. A., and P. Bijma. 2006. Benefits of cooperation between breeding programs in the presence of genotype by environment interaction. J. Dairy Sci. 89:1727-1739. https://doi.org/10.3168/ jds.S0022-0302(06)72241-X.

Mulder, H. A., R. F. Veerkamp, B. J. Ducro, J. A. M. Van Arendonk, and P. Bijma. 2006. Optimization of dairy cattle breeding programs for different environments with genotype by environment interaction. J. Dairy Sci. 89:1740-1752. https://doi.org/10.3168/ jds.S0022-0302(06)72242-1.

Nauta, W. J., R. F. Veerkamp, E. W. Brascamp, and H. Bovenhuis. 2006. Genotype by environment interaction for milk production traits between organic and conventional dairy cattle production in the Netherlands. J. Dairy Sci. 89:2729-2737. https://doi.org/10 .3168/jds.S0022-0302(06)72349-9.

Pedersen, L. D., A. C. Sørensen, M. Henryon, S. Ansari-Mahyari, and P. Berg. 2009. ADAM: A computer program to simulate selective breeding schemes for animals. Livest. Sci. 121:343-344. https://doi .org/10.1016/j.livsci.2008.06.028.

Slagboom, M., L. Hjort $\varnothing$, A. C. Sørensen, H. A. Mulder, J. R. Thomasen, and M. Kargo. 2020. Possibilities for a specific breeding program for organic dairy production. J. Dairy Sci. 103:6332-6345. https://doi.org/10.3168/jds.2019-16900.

Slagboom, M., M. Kargo, A. C. Sørensen, J. R. Thomasen, and H. A. Mulder. 2019. Genomic selection improves the possibility of applying multiple breeding programs in different environments. J. Dairy Sci. 102:8197-8209. https://doi.org/10.3168/jds.2018-15939.

Sundberg, T., L. Rydhmer, W. F. Fikse, B. Berglund, and E. Strandberg. 2010. Genotype by environment interaction of Swedish dairy cows in organic and conventional production systems. Acta Agric Scand. A Anim. Sci. 60:65-73. https://doi.org/10.1080/09064702 .2010 .496003 .

\section{ORCIDS}

Margot Slagboom (ㄷ) https://orcid.org/0000-0002-0296-356X Jørn Rind Thomasen (D) https://orcid.org/0000-0001-7068-0572 